\title{
Exploring Tax Evasion in the Context of Political Uncertainty*
}

\author{
Barbara G. Katz \\ Stern School of Business, New York University \\ 44 W. 4th St., New York, NY 10012 \\ bkatz@stern.nyu.edu; tel: 212998 0865; fax: 2129954218 \\ corresponding author \\ Joel Owen \\ Stern School of Business, New York University \\ 44 W. 4th St., New York, NY 10012 \\ jowen@stern.nyu.edu; tel: 212998 0446; fax: 2129954003
}

November 2012 


\section{Abstract \\ Exploring Tax Evasion in the Context of Political Uncertainty}

We present a model of agents facing the uncertainty of two future forms of government who are able to insure against this uncertainty by hiding funds from taxation. In order to choose whether or not to hide funds from taxation, agents need to know policy choices that each government would make should it come to power. But each government, before it could make its decision, would need to know the choices of the agents who would, for example, produce tax revenues. This informational tension is resolved endogenously. We derive the resulting level of tax evasion in society and the optimal choices made by the potential governments. We examine how changes in governmental structure would affect the level of tax evasion, and how that, in turn, would affect a particular form of capital flight.

JEL Classifications: P37, P26, P27, K42

Key Words: Tax evasion, political uncertainty, economic uncertainty, policy uncertainty, economies in transition 


\section{Introduction}

Between 1990 and winter 2011, thirty-nine incumbents were replaced in a total of fifty-two elections in the eight Central Eastern European countries that joined the European Union in 2004. ${ }^{1}$ These frequent electoral changes, often bringing reversals in ideological orientations, created a climate of political instability as they often resulted in governments pursuing different social and economic policies than their predecessors. $^{2}$ Evidence that economic policy changes have an impact on business decision makers can be found, for example, in the BEEPS II data base. Turning to the broader group of twenty-six economies in transition covered in this study, hundreds of respondents from each of these countries were asked many questions, including the following: How great an obstacle to the operation and growth of your business is economic policy uncertainty? In twenty-two of those countries, more than fifty per cent of the respondents stated that economic policy uncertainty was either a moderate or a major obstacle to the operation and growth of their business. ${ }^{3}$

Of course, the post-Soviet-type economies in transition do not have a monopoly on political uncertainty. In Western Europe, for example, incumbents in Portugal, Spain, France and Greece, to note only four, were even more recently replaced. Indeed, according to a recent paper by Baker, Bloom and Davis (2012), who construct several

\footnotetext{
${ }^{1}$ See Kornai (2006, Table 4) for the list of the thirty governmental turnovers in these countries between 1990 and 2004. These eight economies in transition were the Czech Republic, Estonia, Poland, Latvia, Lithuania, Hungary, Slovakia and Slovenia. Using the same methodology and countries, and extending the time period to March 2011, we find thirty-nine instances in which incumbents were replaced in a total of fifty-two elections.

${ }^{2}$ We offer another comment on political instability, this time relating to Ukraine, a post-Soviet economy in transition. In Ukraine, ahead of the presidential election in January 2010, policy differences among the top contenders included whether or not to change the constitution to return to presidential rule, whether or not to pursue NATO membership, and whether or not to attempt to renegotiate gas prices with Russia.

${ }^{3}$ See BEEPS II Interactive Dataset, EBRD-World Bank, 2002. The question can be found under the heading Governance and Anti-Corruption. We excluded Turkey from the panel of countries, leaving the twenty-six economies in transition.
} 
novel indices of policy uncertainty, policy uncertainty is responsible for a growing proportion of the more general category of economic uncertainty. ${ }^{4}$ Their paper also documents that U.S. policy uncertainty increases at moments of federal elections. Clearly, at most election moments in most countries, there is uncertainty about the policies of the government that will emerge as the victor. In the post-communist period, however, both political instability and divergent ideologies were pervasive features of the economies in transition, and it is for this reason that we set our problem in their context, although the implications of our analysis are applicable more generally.

All the economies in transition suffered immediate drops in output, with real GDP falling for all of them until 1994. Furthermore, only three countries (Poland, Slovenia and Slovakia) had equal or higher real GDP in 1999 than they did in $1989 .{ }^{5}$ The recognition that the economies in transition had outdated capital stocks and production methods, and needed thorough redesign in every area, e.g., economic, legal, political, suggests the scope of the problem that any government in that group of countries had to confront. With no recent tradition of democratic government, and in some cases no tradition of democratic government at all, and with the opportunism that the turmoil and early privatization efforts created, it is not surprising that some of the new governments were more benevolent and focused on improving their countries and others were not. It is in this context that the role of uncertainty becomes especially important to agents in the economies in transition. The uncertainty concerning the various possible future policy choices of the government not only affected the economic decisions of the agents, but also created political pressures in support of different governments. Also during this time, and as a consequence of agents' eco-

\footnotetext{
${ }^{4}$ See the references in this paper for background reading on policy uncertainty and economic outcomes.

${ }^{5}$ See EBRD Transition Reports, various years, and see Campos and Coricelli (2002).
} 
nomic decisions, the economies in transition exhibited generally heightened, albeit varying, levels of tax evasion as well as capital flight. Because of the policy uncertainty during this period, agents were forced to make decisions in the absence of knowing whether the next government would be more or less benevolent, more or less democratic, more or less corrupt, or more or less able and willing to pursue economic growth and infrastructure development.

How would economic agents in an economy in transition have dealt with the significant economic policy uncertainty that they faced? Would this policy uncertainty have induced acts by these agents that would have undermined or impeded the development of stable market-oriented democracies? In this paper, we attempt to answer these questions by investigating the degree to which uncertainty concerning future governmental policy induces tax evasion on the part of agents and how this, in turn, has an impact on economic development.

In our model we imagine agents in a country in an early stage of transition from a planned to a market economy and suppose the transfer of property rights, once held by the state, has already occurred. However, the transition is still in progress, and the nature of the government's future policies is unknown to the agents. In particular, we assume that the agents believe that, due to a variety of reasons, the present government may evolve into either a benevolent government or a corrupt government. Each agent is forced to make economic decisions relating to his firm prior to the knowledge of which government will come into existence. The agent must choose whether or not to shelter some of the firm's funds out of the reach of the tax authorities to compensate for the uncertainty he faces. In choosing whether or not to hide funds from taxation, and how much to hide, the agent needs certain information. We assume that the agent needs to know, among other things, the probability that each government would come into existence and what each of the governments would do if it did come into existence. Knowing these things, each agent 
makes his economic choice, and collectively these choices yield both the tax revenue that would go to the ensuing government, and the level of tax evasion, denoted by the proportion of agents who hide funds from taxation. On the other hand, agents believe that each government, should it come into existence, will optimize its own objectives. These objectives may depend on the level of tax evasion and will be limited by the tax revenues provided by the agents. However, the level of tax evasion level itself will be affected by the decisions of the ensuing government. Thus, the agents need the governments' decisions to solve their problems and the governments need the agents' decisions to solve their problems. The tension between the agents and governments is resolved by endogenizing the probabilities that each government will come into existence, thus bringing these two sets of decisions into accordance. The result of the equilibrium is to produce the level of tax evasion in the society, and should the benevolent government come into existence, the optimal level of investment in infrastructure this government would choose.

To investigate some policy implications of our model, we next explore the impact of changes in the model's parameters on the level of tax evasion and the benevolent government's investment in infrastructure. We then define a specific type of capital flight within the context of our model and show how it would be affected by changes in the model's parameters.

In the context of the literature on the rule of law in transition economies, most models investigating the decisions of agents in transition economies to steal start with a benchmark case in which the agents face a known type of government. The agent optimizes given the existing government, and then the problem is re-solved under the assumption of a different form of government. The agents' decisions in the two situations are then compared. Although agents make decisions in two different contexts, there is no self-awareness on the part of the agents that the government might be of different types. Examples of studies in this tradition include Polishchuk 
and Savvateev (2004), Roland and Verdier (2003), Sonin (2003), and Katz and Owen (2009). A particular type of government, known to the agents, is also assumed in Grossman (1995) and Alexeev, Janeba and Osborne (2004), who focus on "mafias" competing with the state for entrepreneurial rents. Dixit (2004), who suggests a principal-agent model to capture the intent of a government to induce efficiency in society, also assumes a given governmental form, known to the agents. Hoff and Stiglitz (2004) take a different tack by assuming agents face uncertainty regarding the form of government that will arise. They endogenize the probability of occurrence of these governments using a consistency requirement among the agents, and find that the uncertainty of governmental form leads to multiple solutions for the crime level. In their model the agents do not consider an active government in making their choices.

We contribute to the literature on the rule of law in transition by considering the impact of political uncertainty on tax evasion in a broader context than the literature mentioned above. The considerations that the agents face in our model include not only the uncertainty of the future form of government, but also the awareness that their collective decisions have an impact on which government will come into existence, and also on what each government would do if it did come into existence. Taking these awarenesses into account, we derive the level of tax evasion resulting from them. We further examine how changes in this level of tax evasion would be affected by changes in the parameters describing these governments. This leads us to offer certain policy suggestions. We are also able to investigate how changes in a particular type of capital flight would relate to changes in the parameters.

We also contribute to the literature on the role of institutions in transition (for example, Djankov and Murrell (2002), McMillan (2002) and Bevan and Estrin (2004), and to that stressing the importance of institutional arrangements (for example, Shleifer and Vishny (1998), and Acemoglu, Johnson and Robinson (2001, 2002, 
2003)). We add to this literature by showing that different economic outcomes are to be expected when we allow agents to make choices in the face of the uncertainty as to which institutional arrangements will prevail.

We present our model in Section 2. In Section 3 we discuss our results and offer our conclusions.

\section{Model}

\subsection{The Agent's Problem}

We begin by assuming that all agents believe that the present government is in flux and will evolve by the end of the period into one of two possible forms that are known to the agents. We designate the two forms as $\mathrm{G}$ and $\mathrm{B}$. $\mathrm{G}$ is a benevolent government that is concerned with determining its growth rate $r, r \in \mathbb{R}$, so that it maximizes its objective function. That objective function depends on growth and the level of tax evasion (LTE), albeit constrained by its tax revenue and the costs associated with generating growth. $\mathrm{B}$ is a corrupt government that is interested in maximizing the difference between its tax revenue and its costs of maintaining the status quo for its own purposes. While both $\mathrm{G}$ and $\mathrm{B}$ punish tax evasion, B chooses the severity by which it punishes tax evasion in order to maximize its objective function. Thus, at the outset, agents must make decisions in the face of the uncertainty of governmental form, and also with the awareness that their choices will alter the tax revenues and the LTE, and hence the decisions made by the particular government that does come into being. In fact, the ultimate choice of governmental form may itself depend on the collective decisions of the agents.

There is a continuum of expected value maximizing agents $a, a \in[0,1]$, each of whom owns one firm, the value of which is normalized to 1 . Operating a firm honestly might turn out to be unrewarding if $\mathrm{G}$ were to come into existence and, due 
to shortfalls in tax revenue, could only offer a negative growth rate. In this case, it could be beneficial for the agent to hide some of the firm's funds from taxation to insure against this potential loss. On the other hand, should the agent choose to insure against this loss by such hiding of funds and B were to come into existence and the agent were caught hiding funds to avoid taxation, the result could be severe. This motivates us to begin our model by assuming that the agent must decide whether or not to hide funds of the firm from taxation and, if he chooses to hide them, the amount to hide. We let $\tau_{a}, \tau_{a} \in[0,1]$, be the proportion of the firm's funds that agent $a$ chooses to hide from taxation. We assume that the probability that an agent who hides $\tau_{a}$ will be caught is given by $a$. This probability of being caught is invariant to the subsequent form of government.

We first consider the outcomes for the agent should $\mathrm{G}$ come into existence. If agent $a$ chooses to hide $\tau_{a}>0$ proportion of the firm and is not caught, he keeps the hidden funds $\tau_{a}$, and the remainder of the firm, which we assume is honestly run, at the end of the period is worth $(1-t)\left(1-\tau_{a}\right)(1+r)$, where $t, t \in[0,1]$, is the tax rate and $r, r \in \mathbb{R}$, represents the impact of the economic growth generated by $\mathrm{G}$. If agent $a$ is caught, then the agent must return the hidden funds to the government, plus pay a penalty i.e., he must pay back to the government $\tau_{a}(1+\delta), \delta>t$. The part of the firm not shielded from taxation, which we assume to have been run honestly, will be taxed at the rate $t$, and, at the end of the period, will be worth $(1-t)\left(1-\tau_{a}\right)(1+r)$. Thus, agent $a$ retains $(1-t)\left(1-\tau_{a}\right)(1+r)-\delta \tau_{a}$ of the firm at the end of the period. If agent $a$ chooses not to hide funds $\left(\tau_{a}=0\right)$, then the firm will be worth $(1-t)(1+r)$ at the end of the period. Under $\mathrm{G}$, the expected value to agent $a$ of hiding $\tau_{a}>0$ can be written as $E_{1}\left(\tau_{a}\right)=(1-t)(1+r)-\tau_{a}[a(1+\delta)+(1-t)(1+r)-1]$. Under $\mathrm{G}$, the expected value to agent $a$ of hiding $\tau_{a}=0$ is $E_{1}(0)=(1-t)(1+r)$.

We now consider the outcomes for the agent should B come into existence. If agent $a$ chooses to hide $\tau_{a}>0$ and is not caught, he pockets $\tau_{a}$ and the remainder of 
the firm is honestly run and worth $(1-t)\left(1-\tau_{a}\right)$ at the end of the period, where $t$ is the same tax rate as in G. Since B does not create a climate conducive to economic growth, the value of the firm remains constant. If agent $a$ is caught hiding funds, then he is punished by having to pay a penalty $f, f \in\left[t, f_{0}\right]$, where $f_{0} \geq 1+\delta$, on the entire firm to the government. If agent $a$ chooses not to hide funds, then the firm is worth $(1-t)$. Under $\mathrm{B}$, the expected value to agent $a$ of hiding $\tau_{a}>0$ can be written as $E_{2}\left(\tau_{a}\right)=(1-a)(1-t)+a(1-f)+\tau_{a}(1-a) t$. Under B, the expected value to agent $a$ of hiding $\tau_{a}=0$ is $E_{2}(0)=(1-t)$.

To complete the calculation of the expected value for agent $a$, agent $a$ must ascribe a probability to the coming into existence of $\mathrm{G}$ or $\mathrm{B}$. We let $\pi, \pi \in[0,1]$, represent the probability of $\mathrm{G}$ coming into existence, and $(1-\pi)$ the probability of $\mathrm{B}$ coming into existence. Given $\pi$, the expected value to agent $a$ of hiding $\tau_{a}>0, E\left(\tau_{a}\right)=$ $\pi E_{1}\left(\tau_{a}\right)+(1-\pi) E_{2}\left(\tau_{a}\right)$, can be written as

$$
\begin{gathered}
E\left(\tau_{a}\right)=\pi(1-t)(1+r)+(1-\pi)[a(1-f)+(1-a)(1-t)]+ \\
\tau_{a}\{(1-a) t-\pi[a(1-t+\delta)+r(1-t)]\} .
\end{gathered}
$$

Given $\pi$, the expected value to agent $a$ of hiding $\tau_{a}=0$ is $E(0)=\pi E_{1}(0)+(1-$ $\pi) E_{2}(0)$, which can be written as

$$
E(0)=\pi(1-t)(1+r)+(1-\pi)(1-t)
$$

In these expressions, $t$ and $\delta$ are taken to be parameters, but $r$ is to be determined by $\mathrm{G}$ should it come into existence, and $f$ is to be determined by $\mathrm{B}$ should it come into existence. Conditional on $\pi, r$, and $f$, the optimum value of $\tau_{a}$, denoted by $\tau_{a}^{*}$, is summarized in the following proposition. The proof of this proposition and all subsequent propositions can be found in the Appendix. 
Proposition 1. Given $\pi, r$, and $f, \tau_{a}^{*}= \begin{cases}1 & \text { if } a<b(\pi, r, f) \\ 0 & \text { if } a \geq b(\pi, r, f)\end{cases}$ where $b(\pi, r, f)=\frac{t-\pi(1-t) r}{f+\pi(1+\delta-f)}$.

Although agents believe that $r$ and $f$ are to be chosen optimally by $\mathrm{G}$ and $\mathrm{B}$, respectively, the value of $\pi$ should reflect the agents' common view of the probability that $\mathrm{G}$ will come into existence. The way the agents determine $\pi$ depends on the process that will lead to the selection of $\mathrm{G}$ or $\mathrm{B}$, as well as the decisions that these governments would make should they come into existence. In the next section, we address the way in which $\pi$ is endogenized and reevaluate $\tau_{a}^{*}$ using this derived form of $\pi$. (The notation $\tau_{a}^{*}$ will remain when we subsequently insert the optimum values of $\pi, r$, and $f$. )

\subsection{The Conditional Level of Tax Evasion and Endogenization of $\pi$}

We begin by evaluating the conditional LTE. By indexing agent $a$ on the probability that the agent will be caught, we are allowing that some agents are better at hiding funds from taxation than others. How they are distributed in society is assumed to be given by the distribution function $H(a), a \in \mathbb{R}$, where $H(a)=0$ for $a \leq 0, H(a)=1$ for $a \geq 1$, and $H(a)$ is continuous. The LTE conditional on $\pi, r$, and $f$, denoted by $K(\pi, r, f)$, is given by the proportion of agents that hide funds given $\pi, r$, and $f$. It follows from $\mathrm{P} 1$ that $K(\pi, r, f)=\int_{0}^{b(\pi, r, f)} d H(a)$ and $0 \leq K(\pi, r, f) \leq 1$. (Cet. par., $K(\pi, r, f)$ is decreasing in the growth rate $r$.) We now address $\pi$.

The value of $\pi$ must be determined before agents can decide what proportion, if any, of the firm to shelter from the tax authorities. At the outset, agents have no information about the LTE, tax revenues, or government policies, but can decide how they would determine $\pi$ when such information becomes available. Also at the outset, before deciding whether or not to hide funds, we assume all agents prefer higher 
growth rates and lower levels of tax evasion. Since G's objective is to produce higher growth rates and lower levels of tax evasion, we assume that agents would be more supportive of $\mathrm{G}$ the more they perceive $\mathrm{G}$ to be successful in achieving its objectives. That is, we assume that agents perceive $\pi$ to be a function of the growth rate and the LTE, increasing in the first and decreasing in the second. The proportion of honest agents, $1-K(\pi, r, f)$, is decreasing in the LTE and, from the preceding paragraph, it follows that it is increasing in the growth rate. ${ }^{6}$ Thus, this function can serve as a basis for determining $\pi$. We proceed to endogenize $\pi$ by assuming that agents will agree on the value of $\pi$ that satisfies $\pi=1-K(\pi, r, f)$.

We next establish the solution to the equation $\pi=1-K(\pi, r, f)$. To emphasize the conditionality of this solution, we denote it by $\pi(r, f)$ and the resulting conditional LTE as $K(r, f)$.

For the remainder of the model, we set $H(a)=a$ for $a \in(0,1)$. When $H(a)$ is uniform, $K(\pi, r, f)=b(\pi, r, f)$ for $b(\pi, r, f) \in(0,1), K(\pi, r, f)=0$ for $b(\pi, r, f) \leq$ 0 and $K(\pi, r, f)=1$ for $b(\pi, r, f) \geq 1$. Several results hold for other distribution functions as we note in our concluding section.

\section{Proposition 2. Given $r$ and $f$}

(a) If $r \geq t /(1-t)$, then $\pi(r, f)=1$.

(b) If $r<t /(1-t)$, then $\pi(r, f)$ is the unique root in $[0,1)$ satisfying $(f-1-$ $\delta) \pi^{2}-[f-(1+\delta-f)-(1-t) r] \pi+f-t=0$.

Having established the unique root $\pi(r, f)$, we now return to $\mathrm{P} 1$ and evaluate $\tau_{a}^{*}$ using this value. We present the results in the next proposition.

\section{Proposition 3. Given $r$ and $f$}

(a) If $r \geq t /(1-t)$, then $\tau_{a}^{*}=0$ for all $a$.

(b) If $r<t /(1-t)$, then $\tau_{a}^{*}=1$ if $a<1-\pi(r, f)$ and $\tau_{a}^{*}=0$ if $a \geq 1-\pi(r, f)$.

\footnotetext{
${ }^{6}$ As a shorthand, we refer to agents who do not hide funds from taxation as honest.
} 
Having shown the way in which $\pi(r, f)$ and $\tau_{a}^{*}$ depend on $r$ and $f$, agents must now consider the way in which $r$ and $f$ would be chosen by G and B, respectively.

\subsection{Determining Optimum Choices for G and B}

Agents believe $\mathrm{G}$ and $\mathrm{B}$ would determine the optimum values of $r$ and $f$, respectively, if they were to come into being. The agents derive these values by assuming, in turn, that each government would optimize its objective function if it were to come into existence. We begin with G.

If $\mathrm{G}$ were to come into existence, the agents clearly would know this. Thus, the only uncertainty the agents would face when considering how much to hide would be whether or not they would be caught. Using the expected values for agent $a$ given in subsection 2.1 above under the condition of G's existence, agent $a$ would have to evaluate the conditions under which he would hide or not hide funds. These evaluations would lead to the results of the previous subsections when $\pi$ is set equal to 1 . That is, under $\mathrm{G}, \tau_{a}^{*}=1$ if $a \leq[t-(1-t) r] /(1+\delta)$ and 0 otherwise.

Under these circumstances, $b(\pi, r, f)$ becomes $\frac{t-(1-t) r}{1+\delta}$ which we denote by $b_{1}(r)$. The corresponding conditional LTE is denoted by $K_{1}(r)$. It follows that $K_{1}(r)=b_{1}(r)$ for $[t-(1+\delta)] /(1-t)<r<t /(1-t), K_{1}(r)=1$ for $r \leq[t-(1+\delta)] /(1-t)$ and $K_{1}(r)=0$ for $r \geq t /(1-t)$. Thus, the conditional LTE $K_{1}(r)$ is non-increasing in $r$, and therefore the proportion of honest agents is a non-decreasing function of $r$. Since G's objective function is assumed by the agents to be the weighted sum of the growth rate $r$ and the proportion of honest agents, this objective function is an increasing function of the growth rate $r$. Consequently $\mathrm{G}$ will choose $r$ as large as possible subject to its fiscal constraint. This constraint is determined by G's tax revenue $T_{1}(r)$, minus the cost, $C(r)$, associated with producing the growth rate $r$. The tax revenue $T_{1}(r)$ is composed of revenue from the honestly run firms as well as from the penalties levied when those hiding funds from taxation are caught. Thus, 
$T_{1}(r)=\int_{0}^{1}\left[a \tau_{a}^{*}(1+\delta)+t\left(1-\tau_{a}^{*}\right)(1+r)\right] d H$. We next present the explicit form of $T_{1}(r)$.

Proposition 4. $T_{1}(r)=\left\{\begin{array}{cc}t(1+r) & \text { if } r \geq t /(1-t) \\ (1+\delta) / 2 & \text { if } r \leq[t-(1+\delta)] /(1-t) \\ \frac{1+\delta}{2} b_{1}^{2}(r)+t(1+r)\left[1-b_{1}(r)\right] & \text { if } \frac{t-(1+\delta)}{1-t}<r<t /(1-t)\end{array}\right.$ where $b_{1}(r)=\frac{t-(1-t) r}{1+\delta}$.

Because the objective function is increasing in $r$, G will choose $r$ as the largest $r$ such that $T_{1}(r) \geq C(r)$.

If we assume that $C(r)$ is a convex increasing function of $r$ with derivatives $C_{r}(-\infty)=0$ and $C_{r}(\infty)=\infty$, then $\mathrm{G}$ will choose $r$ as the largest $r$ such that $T_{1}(r)=C(r)$. We let $r^{*}$ be the solution.

P4 demonstrates that the tax revenue function is constant up to the value $r=$ $\frac{t-(1+\delta)}{1-t}$ and then becomes a convex parabola, initially decreasing, reaching a minimum and then increasing to the value $r=t /(1-t)$, beyond which it becomes a linear increasing function of $r$. To better understand the shape of $T_{1}(r)$, we stress that this shape is the input to G's considerations in choosing the optimum value of $r$. From this perspective, every agent would hide funds if $b_{1}(r)=1$, i.e., if $r \leq \frac{t-(1+\delta)}{1-t}$. As soon as $r$ exceeds this value, some agents cease to hide funds, that is, they become honest. For each agent who becomes honest, the change in tax revenue that $G$ anticipates is made up of the difference between the new tax paid by the now-honest agent and the loss of penalty payment that that agent, as a former tax evader, would have paid if he had been caught. Since the value of $r$ that immediately precedes $\frac{t-(1+\delta)}{1-t}$ is negative, the tax revenue would be based on a reduced value of the firm, which is less than the amount $\mathrm{G}$ would anticipate if the agent had been dishonest and had been caught. Thus, there would be an anticipated loss of revenues. As $r$ increases and additional agents become honest, this difference remains negative until $r$ is sufficiently large so that the additional tax revenue exceeds the loss of the penalty payments. 
The expression for $T_{1}(r)$ explicitly ties the tax revenue to the LTE, that is, to $K_{1}(r)=b_{1}(r)$. However, this LTE, as argued above, is only the LTE under the assumption that governmental uncertainty has been removed. This LTE is, of course, not the same as the LTE that would result from the behavior of agents facing the uncertainty over governmental form. We now turn to B.

Agents believe B's objective is to choose $f \in\left[t, f_{0}\right]$ to maximize its tax revenue minus the fixed cost of maintaining the status quo. If $\mathrm{B}$ were to come into existence, the agents would clearly know this and agent $a$ would again have to evaluate the conditions under which he would hide or not hide funds from taxation. These evaluations would lead to the results of subsection 2.1 above when $\pi$ is set equal to 0 . That is, under B, $\tau_{a}^{*}=1$ if $a \leq \frac{t}{f}$ and 0 otherwise. Let $b_{2}(f)=\frac{t}{f}$. B's tax revenue is also composed of revenue from honestly run firms as well as from penalties levied when tax evaders are caught. Thus its tax revenue $T_{2}(f)=\int_{0}^{1}\left[f a \tau_{a}^{*}+t\left(1-\tau_{a}^{*}\right)\right] d H$. Evaluating the revenue and subtracting the fixed cost of the status quo, $C(0)$, we have that B's objective function is $t-\frac{1}{2} \frac{t^{2}}{f}-C(0)$. Since this expression is increasing in $f$, B maximizes its objective function by choosing $f^{*}=f_{0}$.

Having determined $r^{*}$ and $f^{*}$, the agents now evaluate $\pi^{*}=\pi\left(r^{*}, f^{*}\right)$. Then, using this value, the agents can calculate $b^{*}=b\left(\pi^{*}, r^{*}, f^{*}\right)$ and subsequently $\tau_{a}^{*}$. Collectively, the choices of the agents yield the LTE $K^{*}=K\left(\pi^{*}, r^{*}, f^{*}\right)=b^{*}$.

\subsection{Example}

To illustrate the results derived above, we now present a special case of the model that brings the two governments under consideration into clearer focus. In this special case, we assume that the penalty chosen by B is the same as that adopted by G, i.e., that $f^{*}=f_{0}=1+\delta$. With this simplifying assumption, each agent is reduced to comparing a growth rate optimally chosen by $\mathrm{G}$ with the status quo maintained by B. 
Since the cost function $C(r)$ is independent of the tax revenue $T_{1}(r)$, then any $r^{*}$ can be chosen in this example by judiciously selecting the cost function. The most interesting results derived in the propositions above occur when $r<t /(1-t)$. Thus, in this example, we set $r^{*}=\alpha^{*} \frac{t}{1-t}$ where $\alpha^{*}<1$.

To further simplify the notation of this example, we write $1+\delta$ in units of the tax rate, i.e., we set $f^{*}=f_{0}=1+\delta=n t$ with $n>1$. With this assumption and change of notation, the equation of $\pi^{*}$ in P2 part (b) becomes linear and yields $\pi^{*}\left(r^{*}, f^{*}\right)=\frac{n-1}{n-\alpha^{*}}$. Using the definition of $b(\pi, r, f)$ given in P1 and the fact that $K(\pi, r, f)=b(\pi, r, f)$ in our model, it follows for this example that $K^{*}\left(\pi^{*}, r^{*}, f^{*}\right)=$ $b^{*}\left(\pi^{*}, r^{*}, f^{*}\right)=\frac{1-\alpha^{*}}{n-\alpha^{*}}$. Finally, from P1, $\tau_{a}^{*}=1$ if $a<\frac{1-\alpha^{*}}{n-\alpha^{*}}$ and $\tau_{a}^{*}=0$, otherwise.

For this example, no matter which $r^{*}$ is chosen by G, neither government will ever be the unambiguous choice of the agents, i.e., $0<\pi^{*}\left(r^{*}, f^{*}\right)<1$, for $r^{*}>$ $-\infty$. Reflecting this observation is the fact that there will always be some LTE, i.e., $K^{*}\left(\pi^{*}, r^{*}, f^{*}\right)>0$, no matter the value of $r^{*}$. This is also shown in the value of $\tau_{a}^{*}$ where there are always agents for whom $\tau_{a}^{*}=1$.

Now consider an increasing sequence of $n$ values and, correspondingly, a sequence of cost functions that, for each of these $n$ values, yields $r^{*}=0$, i.e., $\alpha^{*}=0$. This produces a sequence of $\pi^{*}$ and $K^{*}$ values. The sequence of $\pi^{*}$ values is strictly increasing in $n$ and the sequence of $K^{*}$ values is strictly decreasing in $n$. When $r^{*}=0$, the growth rate is the same for $\mathrm{G}$ and $\mathrm{B}$, yet $\pi^{*}=1 / 2$ only if $n=2$. The reason for this is that $K^{*}=1 / 2$ only for $n=2$. The value of $\pi^{*}$ will increase or decrease from $1 / 2$ as $K^{*}$ decreases or increases from $1 / 2$. This is consistent with our assumption that agents, before having made their choices, consider both growth and tax evasion in their determination of $\pi$. These properties of the example can be shown to hold in the general case.

The conclusions of the last paragraph do not hold if we keep the cost function fixed and increase the value of $n$, since in this case, $T_{1}(r)$ will change and, as a consequence, 
$r^{*} \neq 0$. Thus, we need a different approach to explore the consequences of changing parameter values in our model. We address this in the next section.

\subsection{Comparative Statics: $r^{*}$ and $f^{*}$}

In order to investigate how changes in the fundamental parameters $\delta, t$, and $f_{0}$ would affect the LTE or the support the agents might provide for a particular government, we must first analyze how the government's choices of $r^{*}$ and $f^{*}$, respectively, change with these parameters. In what follows, the subscript $\theta$ under a function denotes partial differentiation with respect to the subscripted variable.

Proposition 5. For $\theta=\delta$, t, or $f_{0}, \operatorname{sign} \frac{d r^{*}}{d \theta}=\operatorname{sign} T_{1 \theta}\left(r^{*}\right)$.

Using P4, we differentiate $T_{1}(r)$ and, depending in which region $r^{*}$ is found, we can determine the sign of $\frac{d r^{*}}{d \theta}$ as required by $\mathrm{P} 5$. We focus on the sign since, as can be seen in the proof of $\mathrm{P} 5$, the magnitude of $\frac{d r^{*}}{d \theta}$ depends on the reciprocal of $T_{1 \theta^{*}}-C_{r^{*}}$ and therefore can have any value depending on the specific specifications of the model. This leads to the next proposition.

Proposition 6. (a) $\frac{d r^{*}}{d \delta} \geq 0$ for all $r^{*}$ except for $r^{*} \in\{[t-(1+\delta)] /(1-t),-t /(1+t)\}$ where $\frac{d r^{*}}{d \delta}<0$.

(b) $\frac{d r^{*}}{d t} \geq 0$ for all $r^{*}$ except for $r^{*} \in\{[t-(1+\delta)] /(1-t),-1\}$ and for $r^{*} \in$ $\{\min [(1+\delta-t) / t, t /(1-t)], t /(1-t)\}$ where $\frac{d r^{*}}{d t}<0$.

(c) $\frac{d r^{*}}{d f_{0}}=0$ for all $r^{*}$.

(d) $\frac{d f^{*}}{d t}=0, \frac{d f^{*}}{d \delta}=0$ for all $f_{0}$ except when $f_{0}=1+\delta$ where $\frac{d f^{*}}{d \delta}>0$.

(e) $\frac{d f^{*}}{d f_{0}}>0$.

In parts (a)-(c) of $\mathrm{P} 6$, we consider changes in $r^{*}$ with respect to changes in the parameters. As such, we are considering the agents' problem from the view point of G having come into existence. In part (a) we show that generally an increase in the 
penalty for tax evasion allows G1 to increase $r^{*}$. There is, however, an exception to this, that occurs when the $r^{*}$ that $\mathrm{G}$ can afford falls into the negative range given in part (a). The attempt to increase the penalty in this range reduces $r^{*}$ since raising the penalty has two impacts. One is to increase revenue and the other is to decrease the LTE. In this range, an agent who was hiding funds and was caught and who would have paid $\mathrm{G}$ the additional amount $d \delta$ is now honest, and pays the tax of $t\left(1+r^{*}\right)$. Since $r^{*}$ here is negative, part (a) establishes that the net effect to $\mathrm{G}$ is negative.

In part (b) of $\mathrm{P} 6$, we find that an increase in the tax rate generally allows $\mathrm{G}$ to choose a large $r^{*}$. This is not true when $r^{*} \in\{[t-(1+\delta)] /(1-t),-1\}$, where for similar reasons as discussed in the paragraph above, $\frac{d r^{*}}{d \delta}<0$. However, in part (b), there is a second possible interval, $\left.r^{*} \in\{\min [1+\delta-t] / t, t /(1-t)), t /(1-t)\right\}$ where $\frac{d r^{*}}{d t}<0$. This interval vanishes unless $[(1+\delta-t) / t]<[t /(1-t)]$ or when $t>/[(1+\delta) /(2+\delta)]$. For $r^{*}>0$, increasing $t$ has two effects, increasing revenue and increasing the LTE. Thus, an agent who was hiding none of the firm's funds from taxation, and would have paid the additional amount of taxes $d t\left(1+r^{*}\right)$, would now hide funds and, if caught, pay $1+\delta$. Depending on the size of $r^{*}$, the net effect of this change could be either positive or negative, but part (b) establishes the relationship between $t$ and $\delta$ that causes the net effect on $\mathrm{G}$ to be negative. In sum, parts (a) and (b) imply that the normal intuition that raising taxes, whether on tax evaders $(\delta)$ or on honest agents $(t)$, will increase revenues and therefore the options of $\mathrm{G}$, is correct. However, parts (a) and (b) also imply that this intuition is incorrect in extreme cases as specified in the proposition. In these extreme cases, the raising of taxes could worsen the situation. Since $r^{*}$ does not depend on $f_{0}$, part (c) follows.

In parts $(\mathrm{d})$ and (e) agents are considering the problem from the viewpoint of $\mathrm{B}$ having come into existence. Since $f^{*}=f_{0}$, parts (d) and (e) follow.

Having established how $r^{*}$ changes with changes in the parameters of the model, we next examine how the LTE would correspondingly change. Then we introduce 
the concept of a specific type of capital flight into our model and investigate how it would change with parametric changes.

\subsection{Comparative Statics: LTE and a type of Capital Flight}

Because agents are uncertain as to the future form of government and its policies, hiding funds from taxation may occur and, as we showed above, this leads to a LTE of $K^{*}$. Of the funds hidden from taxation, some were not recaptured by the government and remained undetected. Below, we will suppose that these undetected hidden funds form the basis of capital flight. Of course capital flight can occur for any number of reasons, most beyond the scope of this model, and need entail no illegal action. Furthermore, tax evasion is not necessary for capital flight. However, to facilitate our discussion of the use to which the undetected hidden funds might be put in the context of our model, we will make reference to these undetected hidden funds as the basis of capital flight, recognizing that this is an over-simplification of a complex topic. In this section we are concerned with how the LTE changes with changes in our parameters $\delta, t$ and $f_{0}$, and, subsequently, with the impact of changes in the LTE on what we refer to as capital flight. We begin by studying the LTE.

Proposition 7. Under P6,

(a) $\frac{d K^{*}}{d \delta}<0$ if $r^{*} \notin\{[t-(1+\delta)] /(1-t),-t /(1+t)\}$.

(b) There exists a constant $r_{0}$ with $r_{0}<-1$ such that $\frac{d K^{*}}{d t}>0$ if $r^{*}$ is either $\left\{\max \left[r_{0},(t-(1+\delta)) /(1-t)\right],-1\right\}$ or $\left.\{\min [(1+\delta-t) / t), t /(1-t)], t /(1-t)\right\}$. When $r^{*}$ is in neither interval, sign $\frac{d K^{*}}{d t}$ can be positive or negative depending on parameter values.

(c) $\frac{d K^{*}}{d f_{0}}<0$

The function $K^{*}=b^{*}$ depends on $\delta$ in possibly four ways: through $\pi^{*}$, through $r^{*}$, through $f^{*}$, and directly through $\delta$. Thus, the sign of $\frac{d K^{*}}{d \delta}$ must reconcile the 
contributions of each of these four ingredients. We interpret part (a) first. Although some of these ingredients are of opposite signs, when $r^{*} \notin\{[t-(1+\delta)] /(1-t),-t /(1+$ t)\} their sum is always negative. Marginally, increasing $\delta$ causes a decrease in the LTE. An agent who had been a tax evader and would have provided a penalty to $\mathrm{G}$ if caught, would now be honest and would pay taxes. The difference of these two payments to $\mathrm{G}$ is what $\mathrm{G}$ gains as $\delta$ is increased. Usually, the tax contribution is larger than the penalty contribution from the caught tax evader, yielding an increase in revenue. Thus, both an increase in $\delta$ and an increase in revenue produce a decline in the LTE. However, when $r^{*}$ is in the interval $\{[t-(1+\delta)] /(1-t),-t /(1+t)\}$, the net effect of these two payments is negative, causing $r^{*}$ to decline. The effect of this is marginally to increase the LTE. Thus, there are two opposing forces, one to increase and one to decrease the LTE. The reconciliation of these two forces here depends on the parameter values. Thus, if one were considering reducing tax evasion by increasing the penalty for hiding funds from taxation, it would be important to take into consideration the state of economic development to avoid worsening the situation. In particular, in those economies very negative growth rates (for example, some of the economies in transition during specific periods, or otherwise, for example, Greece during the euro crisis), controlling tax evasion by increasing penalties might be counter-productive.

We now turn to part (b). Let $r^{*}$ in part (b) be in the interval $\left\{\max \left[r_{0},(t-(1+\right.\right.$ $\delta)) /(1-t)],-1\}$. Examining the form of $K^{*}=b^{*}$, a marginal increase in $t$ causes $b^{*}$ to increase. Also, from P6, we showed that an increase in $t$ causes $r^{*}$ to fall, marginally causing $b^{*}$ to rise. Thus, unambiguously, the LTE rises. In the positive interval, if it exists, again the marginal effect of $t$ is to increase the LTE. From P7, since $r^{*}$ declines with $t$, this latter effect also causes $b^{*}$ to increase and thus unambiguously the LTE increases with $t$. Outside these two intervals, the marginal effect on $t$ and its consequences on $r^{*}$ yield opposite effects on the total LTE that can only be resolved 
with knowledge of the particular parametric values. The difficulty of assigning a sign to $\frac{d K^{*}}{d t}$ for most values of $r^{*}$ stems from the assumption of convenience that we made at the outset that $t$ is the common tax rate for both $\mathrm{G}$ and B. Since $f_{0}$ enters $b^{*}$ only through $f^{*}$ and since $b^{*}$ declines with $f^{*}$, part (c) follows.

In the discussion following $\mathrm{P} 7$, the contribution of $\pi^{*}$ to the change in $K^{*}$ was not mentioned. Since at optimality, $\pi^{*}=1-K^{*}, \pi^{*}$ was absorbed into $K^{*}$ in this discussion.

We next assume that a portion of the undetected hidden funds leave the country as a type of capital flight. For ease of presentation, in what follows we assume that capital flight, denoted by $F$, is equal to the total undetected hidden funds.

From earlier discussions, $F=\int_{0}^{1}(1-a) \tau_{a}^{*} d H=\int_{0}^{b^{*}}(1-a) d H=b^{*}-\frac{b^{*^{2}}}{2}=\frac{1-\left[1-K^{*}\right]^{2}}{2}$ if $0<b^{*} \leq 1$ and $F=0$ otherwise. This enables us to describe changes in $F$ in terms of changes in the LTE which we do next.

Proposition 8. For $\theta=\delta, t$, or $f_{0}$,

$$
\frac{d F}{d \theta}=\left\{\begin{array}{cc}
0 & \text { if } r^{*} \geq t /(1-t) \\
\left(1-K^{*}\right) \frac{d K^{*}}{d \theta} & \text { if } r^{*}<t /(1-t)
\end{array} .\right.
$$

P8 establishes the direct connection between our definition of capital flight and the LTE and, in particular, how changes in the one induce changes in the other. When $r^{*}>t /(1-t)$, all agents are honest, the LTE is zero, and small changes in $\delta$, $t$, and $f_{0}$, cause no change in capital flight. When $r^{*} \leq(t / 1-t)$, changes in capital flight will occur and depend on the LTE and its derivative. We next ascertain the direction of this change.

Proposition 9. Let $r^{*}<t /(1-t)$, then

(a) $\frac{d F}{d \delta}<0$ if $r^{*} \notin\{[t-(1+\delta)] /(1-t),-t /(1+t)\}$.

(b) $\frac{d F}{d t}>0$ if $r^{*}$ is in either $\left\{\max \left[r_{0},[t-(1+\delta)] /(1-t)\right],-1\right\}$ or $\{\min [(1+\delta-$ $t) / t, t /(1-t)], t /(1-t)\}$ where $r_{0}$ is given in P7. When $r^{*}$ is in neither interval, the sign of $\frac{d F}{d t}$ can be positive or negative, depending on the parameters. 
(c) $\frac{d F}{d f_{0}}<0$.

Since $\operatorname{sign} \frac{d F}{d \theta}=\operatorname{sign} \frac{d K^{*}}{d \theta}$, the discussion following $\mathrm{P} 7$ is pertinent to all of the results of P9. It is worth noting again that an attempt to control capital flight by raising the penalty on agents hiding funds from taxation might fail if the attempt is made in an economy with a sufficiently negative growth rate. Similarly, a rise in the tax rate can also have undesired consequences on capital flight depending on the state of the economy.

\section{Discussion and Conclusions}

We presented a model of agents facing political uncertainty who were able to insure against this uncertainty by hiding funds from taxation. By allowing agents this choice, the LTE in society emerged and dominated the discussions of all of the features of the model. Not only did the LTE have the usual interpretation, but it also was a major factor in determining tax revenues that the potential governments could expect. As a consequence, the LTE also became an important factor in the optimum choices of the governments. Our model suggests that in a world in which such tax evasion options exist, ignoring them may bias conclusions and policy implications.

A feature of our model worth noting is that of asymmetric information. When agents confront the decision to hide funds from taxation, they face two sources of uncertainty: Not knowing which government will come into being and not knowing if they would be caught if they hid funds from taxation. Based on these two sources of uncertainty, the model determined the LTE that would ensue. But there were two other levels of tax evasion that were also involved in the model. When the agents assumed that $\mathrm{G}$, having come into being, optimized its objective function subject to its fiscal constraint, G needed to anticipate the LTE since this level was part of its objective function, as well as a contributor to its tax revenue. But agents 
facing G, assuming it had come into existence, have only one source of uncertainty to contend with, namely, the uncertainty of being caught when hiding funds from taxation. Therefore, the LTE as perceived by G in this circumstance is different from that as perceived by the agents before imagining $\mathrm{G}$ had come into existence. Similarly, another LTE was determined when agents imagined B had come into existence. Since the agents need to know the optimum choices of the governments before they can make their own choices, it follows that the actual tax revenue produced by the agents would not be the tax revenue used by the governments to optimize their choices, and that the levels of tax evasion produced by the agents would not be the ones used by the governments to optimize their choices. While, for example, it is always true that the LTE produced by the agents under full uncertainty is between those conceived of by $\mathrm{G}$ and $\mathrm{B}$, the relative sizes of these latter two levels of tax evasion depend on information that is not available to either government. The implications of these discrepancies remain to be analyzed.

In the model, two assumptions were made concerning the ways the set of agents select the subjective probability, $\pi$, that $\mathrm{G}$ will come into existence. First, we assumed that the more agents believed that $\mathrm{G}$ could obtain its objectives, the higher $\pi$ would be, and conversely. The second assumption was to set $\pi$ equal to a particular function that was shown to move in accordance with G's objectives. These two assumptions permitted us to endogenize the value of $\pi$ and to proceed to analyze the full model. Obviously, the particular function we chose might have been different, but if it still satisfied our first assumption, then we believe that the results would not have substantially changed.

The importance of the LTE in our model is underscored by its complexity. We showed that, among other things, the LTE depended on $\pi^{*}$, the agreed upon probability that $\mathrm{G}$ would come into existence, $r^{*}$, the growth rate chosen by $\mathrm{G}$, and $f^{*}$, the penalty rate chosen by B. All of these optimized values depended on the perceived 
behavior of groups of agents and the structural values defining each government. Because of the importance of the LTE in policy-making, governments might consider controlling this level by altering the structural values. The result of our study shows that changes in these values do not necessarily yield desirable changes in the LTE. That is, due to the complexity just mentioned, changes in the structural values can lead to unintended changes in the LTE. Also, since what we term capital flight in our model is directly related to the LTE, it follows that attempts to control it by altering structural values should be done in light of these possible unintended consequences.

Our model was formulated to explain some of the challenges faced by agents in the economies in transition. To that end, we derive, among other things, the LTE in a particular stylized country. But how can the model speak of different levels of tax evasion in different countries more generally? We answer this question by thinking of an economy as made up of three elements: First, the structural form of possible future governments faced by the agents, second, the distribution of agents' skills $H(a)$, and third, the cost $C(r)$ in the economy of achieving a growth rate $r$. These elements need to be chosen to match the characteristics of a given country. For example, the two possible governmental outcomes could involve less benevolent options and more draconian options. Also, a country might initially be more corrupt than another and thus $H(a)$ might increase faster for the more corrupt country. Finally, one country could initially be poorer than another, or less able to attract or be granted funds, implying that its cost of improving the infrastructure would be larger. Specifying these values for a given country, we would proceed in the development of the corresponding model. However, depending on how far afield these elements are from those assumed in our model, the development itself might become more complex. For example, if $H(a)$ were specified not to be uniform but to be convex, the development would go through essentially unchanged. But, more complicated specifications of these distribution might lead to multiple solutions and 
the problems associated with those solutions. However, by changing the form of these three elements, singly or in combination, while maintaining the essential argument developed in this paper, we are able to offer a partial explanation for the differences in crime levels, capital flight and growth rates in the economies in transition. We hope that others might take an interest in our approach and use it as a basis for empirical studies.

\section{Appendix}

Proof of P1. Agent $a$ will choose $\tau_{a}^{*}=\arg \max \left[E(0), \max _{0<\tau_{a} \leq 1} E\left(\tau_{a}\right)\right]$. (A tie in expected values is assumed to lead agent $a$ to be honest.) Since $E\left(\tau_{a}\right)$ is linear in $\tau_{a}$, agent $a$ will choose $\tau_{a}=0$ or $\tau_{a}=1$. The intercept of this line is less than $E(0)$. This follows by setting $\tau_{a}=0$ in equation 2.1, and comparing that result to $E(0)$, noting that $f \in\left[t, f_{0}\right]$. Thus, a necessary and sufficient condition that $\tau_{a}^{*}=1$ is $E\left(\tau_{a}=1\right)-E(0)>0$. Using equations 2.1 and 2.2 ,

$$
\begin{aligned}
E\left(\tau_{a}=\right. & 1)-E(0)=(1-\pi)[a(1-f)+(1-a)(1-t)-(1-t)]+t \\
& -\pi(1-t) r-a[t+(1+\delta-t) \pi] \\
= & t-\pi(1-t) r-a[t+(1+\delta-t) \pi+(1-t)(1-\pi)] \\
= & t-\pi(1-t) r-a[f+(1+\delta-f) \pi)] .
\end{aligned}
$$

This last expression is positive if and only if $a<\frac{t-\pi(1-t) r}{f+\pi(1+\delta-f)}$. Thus, $\tau_{a}^{*}=1$ if the last inequality holds and $\tau_{a}^{*}=0$ otherwise.

Proof of P2. Let $r=t /(1-t)$. Then $b(\pi, r, f)=\frac{t(1-\pi)}{f+\pi(1+\delta-f)} \geq 0$. Substituting $b(\pi, r, f)$ for $K(\pi)$ in the equation $\pi=1-K(\pi)$ yields the equation $\pi=1-\frac{t(1-\pi)}{f+\pi(1+\delta-f)}$ which has $\pi=1$ as its solution. For $r>t /(1-t), b(\pi, r, f)$ is negative at $\pi=1$. Thus, 
$K(\pi)=0$ at $\pi=1$ so that $\pi=1-K(\pi)$ has the solution $\pi=1$. This establishes part (a).

To show part (b), we note that for $r<t /(1-t), b(\pi, r, f)>0$. Substituting $b(\pi, r, f)=\frac{t-\pi(1-t) r}{f+\pi(1+\delta-f)}$ for $K(\pi)$ in the equation $\pi=1-K(\pi)$, multiplying through by $f+\pi(1+\delta-f)$ and collecting terms we get that $\pi(r, f)$ must satisfy

$$
(f-1-\delta) \pi^{2}-[f-(1+\delta-f)-(1-t) r] \pi+f-t=0 .
$$

This parabola equals $f-t$ at $\pi=0$ and equals $-[t-(1-t) r]$ at $\pi=1$. Since $f \in\left[t, f_{0}\right], f-t \geq 0$. Since $r<t / 1-t,-[t-(1-t) r]<0$. Thus, the parabola has a unique root in $[0,1]$

Proof of P3. Part (a): If $r \geq t /(1-t)$, then from P2, $\pi(r, f)=1$ and $b[\pi(r, f), r, f] \leq 0$. Thus, for all $a \in[0,1], a \geq b[\pi(r, f), r, f]$ and by P1, $\tau_{a}^{*}=0$ for all $a$.

Part (b): If $r<t /(1-t)$, then $0 \leq 1-\pi(r, f)=b[\pi(r, f), r, f] \leq 1$. Substituting into $\mathrm{P} 1$ yields the result.

Proof of P4. From G's perspective, agent $a$ chooses $\tau_{a}^{*}=1$ iff $a<b_{1}(r)$, and $\tau_{a}^{*}=0$ otherwise. Since $b_{1}(r) \leq 0$ for $r \geq t /(1-t)$, all agents are honest in this range. Because they are honest, they each pay full taxes $t(1+r)$. Since $b_{1}(r) \geq 1$ for $r \leq \frac{t-(1+\delta)}{1-t}$, all agents are tax evaders in this range. Because all agents are tax evaders, agent $a$ is caught with probability $a$ and pays $(1+\delta)$. Collectively, they pay $\int_{0}^{1} a(1+\delta) d H=\frac{1+\delta}{2}$. For $\frac{t-(1+\delta)}{1-t}<r<t /(1-t), 0<b_{1}(r)<1$. Thus, 


$$
\begin{aligned}
T_{1}(r) & =\int_{0}^{1} a \tau_{a}^{*}(1+\delta) d H+\int_{0}^{1} t\left(1-\tau_{a}^{*}\right)(1+r) d H \\
& =\int_{0}^{b_{1}(r)} a(1+\delta) d H+\int_{b_{1}(r)}^{1} t(1+r) d H \\
& =\frac{(1+\delta)}{2} b_{1}^{2}(r)+t(1+r)\left[1-b_{1}(r)\right] .
\end{aligned}
$$

Proof of P5. $r^{*}$ is the largest value of $r$ satisfying $T_{1}(r)-C(r)=0$. Thus, $T_{1}\left(r^{*}\right)-C\left(r^{*}\right)=0$. The parameter $\theta\left(\theta=\delta, t\right.$, or $\left.f_{0}\right)$ appears in $T_{1}\left(r^{*}\right)$ in two ways, through $r^{*}$ and directly through $\theta$. The parameter $\theta$ appears in $C\left(r^{*}\right)$ through $r^{*}$ only. Therefore, by differentiation, $T_{1 r^{*}} \frac{d r^{*}}{d \theta}+T_{1 \theta}-C_{r^{*}} \frac{d r^{*}}{d \theta}=0$ or $\left(T_{1 r}-C_{r^{*}}\right) \frac{d r^{*}}{d \theta}=-T_{1 \theta}$. Since $r^{*}$ is the largest $r$ satisfying this equation, the slope of the cost function must exceed that of the tax revenue function at $r^{*}$. The result follows.

\section{Proof of P6.}

Part (a). For the left interval $r^{*} \leq \frac{t-(1+\delta)}{1-t}, T_{1}\left(r^{*}\right)=\frac{1+\delta}{2}$. Applying the result of $\mathrm{P} 5, \frac{d r^{*}}{d \delta}=1 / 2$. For the middle interval $\frac{t-(1+\delta)}{1-t} \leq r^{*} \leq t /(1-t), T_{1}\left(r^{*}\right)=\frac{(1+\delta)}{2} b_{1}^{2}\left(r^{*}\right)+$ $t\left(1+r^{*}\right)\left[1-b_{1}\left(r^{*}\right)\right]$. Then, $T_{1 \delta}\left(r^{*}\right)=\frac{b_{1}^{2}\left(r^{*}\right)}{2}+\left[(1+\delta) b_{1}\left(r^{*}\right)-t\left(1+r^{*}\right)\right] b_{1 r^{*}}\left(r^{*}\right)$. Since $b_{1}\left(r^{*}\right)=\frac{t-(1-t) r^{*}}{1+\delta}$, we have $T_{1 \delta}\left(r^{*}\right)=\frac{1}{2}\left[\frac{t-(1-t) r^{*}}{1+\delta}\right]^{2}-\left[t-(1-t) r^{*}-t\left(1+r^{*}\right)\right] \frac{t-(1-t) r^{*}}{(1+\delta)^{2}}=$ $\frac{t-(1-t) r^{*}}{2(1+\delta)^{2}}\left[t-(1-t) r^{*}+2 r^{*}\right]$

$=\frac{t-(1-t) r^{*}}{2(1+\delta)^{2}}\left[t+r^{*}(1+t)\right]$. Thus, $\frac{d r^{*}}{d \delta}<0$ if $\frac{t-(1+\delta)}{1-t} \leq r^{*}<-t /(1+t)$ and $\frac{d r^{*}}{d \delta} \geq$ for $-t /(1+t) \leq r^{*} \leq t /(1-t)$. In the right interval, $r^{*}>t /(1-t), T_{1}\left(r^{*}\right)=t\left(1+r^{*}\right)$ and $\frac{d r^{*}}{d \delta}=0$. Thus, looking over all regions, $\frac{d r^{*}}{d \delta}<0$ only if $\frac{t-(1+\delta)}{1-t} \leq r^{*} \leq-t(1+t)$ and part (a) follows.

Part (b). In the left interval $T_{1}\left(r^{*}\right)=\frac{(1+\delta)}{2}$ so $\frac{d r^{*}}{d t}=0$. In the middle interval

$$
\begin{aligned}
& T_{1 t}\left(r^{*}\right)=\left(1+r^{*}\right)\left[\left(1-b_{1}\left(r^{*}\right)\right]+\left[(1+\delta) b_{1}\left(r^{*}\right)-t\left(1+r^{*}\right)\right] b_{1 t}\left(r^{*}\right)\right. \\
& \quad=\frac{1+r^{*}}{1+\delta}\left[1+\delta-t+(1-t) r^{*}\right]-\left[r^{*}\right] \frac{1+r^{*}}{1+\delta}=\frac{1+r^{*}}{1+\delta}\left[1+\delta-t\left(1+r^{*}\right)\right]
\end{aligned}
$$


$=\left(1+r^{*}\right)-\frac{t}{1+\delta}\left(1+r^{*}\right)^{2}$. This quadratic is zero at $r^{*}=-1$ and at $r^{*}=\frac{1+\delta-t}{t}$ and is positive in between. Thus, $\frac{d r^{*}}{d t} \geq 0$ for $r^{*}$ in $\left\{-1, \min \left[\frac{1+\delta-t}{t}, \frac{t}{1-t}\right]\right\}$ and is negative for $r^{*} \operatorname{in}\left\{\frac{[t-(1+\delta)]}{1-t},-1\right\}$. In the right interval $T_{1}\left(r^{*}\right)=t\left(1+r^{*}\right)$ so $\frac{d r^{*}}{d t}>0$ here. Thus, over all regions, $\frac{d r^{*}}{d t} \geq 0$ everywhere except for $r^{*} \in\left\{\frac{[t-(1+\delta)]}{1-t},-1\right\}$ and for $r^{*} \in\left\{\min \left[\frac{1+\delta-t}{t}, \frac{t}{1-t}\right]\right\}$. This establishes part (b).

Part (c) follows since $r^{*}$ does not depend on $f_{0}$.

Part (d) The first part of part (d) follows since $f^{*}$ does not depend on $t$. If $f_{0} \neq 1+\delta$, then $f^{*}$ does not depend on $\delta$ and $\frac{d f^{*}}{d \delta}=0$. If $f_{0}=1+\delta$, then $\frac{d f^{*}}{d \delta}=1>0$ and part (d) follows.

Part (e) follows since $f^{*}=f_{0}$

Proof of P7. The LTE $K^{*}=K^{*}\left[\pi^{*}, r^{*}, f^{*}, \theta\right]$ where $\theta=\delta, t$ or $f_{0}$. Thus, $\frac{d K^{*}}{d \theta}=$ $K_{\pi^{*}}^{*} \frac{d \pi^{*}}{d \theta}+K_{r^{*}}^{*} \frac{d r^{*}}{d \theta}+K_{f^{*}}^{*} \frac{d f^{*}}{d \theta}+K_{\theta}$. As was shown in the proof of P2, for $r^{*}>t /(1-t)$, $b^{*}<0$, and $K^{*}=0$. It follows that when $r^{*}>t /(1-t), \frac{d K^{*}}{d \theta}=0$ for $\theta=\delta, t$ or $f$. We now consider the case when $r^{*} \leq t /(1-t)$. Since $\pi^{*}=1-K^{*}$, we have $\frac{d \pi^{*}}{d \theta}=-\frac{d K^{*}}{d \theta}$. Substituting this expression above, we have $\left(1+K_{\pi^{*}}^{*} \frac{d K^{*}}{d \theta}=K_{r^{*}}^{*} \frac{d r^{*}}{d \theta}+K_{f^{*}}^{*} \frac{d f^{*}}{d \theta}+K_{\theta}^{*}\right.$. As a result of our assumption that $H(a)$ is uniform, $K^{*}=b^{*}$, and the last expression can be written as $\left(1+b_{\pi^{*}}^{*}\right) \frac{d K^{*}}{d \theta}=b_{r^{*}}^{*} \frac{d r^{*}}{d \theta}+b_{f^{*}}^{*} \frac{d f^{*}}{d \theta}+b_{\theta}^{*}$. We first show that $\left(1+b_{\pi^{*}}^{*}\right)>0$. Since $f^{*}=f_{0} \geq 1+\delta$ and $r^{*}<t /(1-t), 1+b_{\pi^{*}}^{*}=1+\frac{t\left(f^{*}-1-\delta\right)-f^{*}(1-t) r^{*}}{\left[f^{*}-\pi\left(f^{*}-1-\delta\right)\right]^{2}} \geq$ $1+\frac{t\left(f^{*}-1-\delta\right)-f^{*} t}{\left[f^{*}\right]^{2}}=1-\frac{t(1+\delta)}{\left[f^{*}\right]^{2}}>0$. So we have that $\operatorname{sign} \frac{d K^{*}}{d \theta}=\operatorname{sign}\left[b_{r^{*}}^{*} \frac{d r^{*}}{d \theta}+b_{f^{*}}^{*} \frac{d f^{*}}{d \theta}+b_{\theta}^{*}\right]$. We use this expression in the following evaluations.

We first note that since $b^{*}=\frac{t-\pi^{*}(1-t) r^{*}}{f^{*}(1-\pi)+\pi^{*}(1+\delta)}$ it follows that $b_{r^{*}}^{*}<0, b_{f^{*}}^{*}<0$, and $b_{\delta}^{*}<0$. Now let $\theta=\delta$. Then $\operatorname{sign} \frac{d K^{*}}{d \theta}=\operatorname{sign}\left[b_{r^{*}}^{*} \frac{d r^{*}}{d \delta}+b_{f^{*}}^{*} \frac{d f^{*}}{d \delta}+b_{\delta}^{*}\right]$. Since $b_{r^{*}}^{*}, b_{f^{*}}^{*}$, and $b_{\delta}^{*}$ are all negative, all terms would be negative if $\frac{d r^{*}}{d \delta}$ and $\frac{d f^{*}}{d \delta}$ are non-negative. From P6, $\frac{d r^{*}}{d \delta}$ is non-negative everywhere except for $r^{*} \in\left\{\frac{t-(1+\delta)}{1-t},-\frac{t}{1-t}\right\}$ and $\frac{d f^{*}}{d \delta}$ is non-negative everywhere. Thus part (a) follows.

Now let $\theta=t$. Then $\operatorname{sign} \frac{d K^{*}}{d t}=\operatorname{sign}\left[b_{r^{*}}^{*} \frac{d r^{*}}{d t}+b_{f^{*}}^{*} \frac{d f^{*}}{d t}+b_{t}^{*}\right]$. From P6, we have $\frac{d f^{*}}{d t}=0$. Differentiating $b^{*}$ with respect to $t$ shows that $b_{t}^{*}$ is a positive multiple of 
$1+\pi^{*} r^{*}$ which is positive at $r^{*}=-1$ and since from P2, $\pi^{*}<1$, there will exist an

$r_{0}<-1$ such that $1+\pi^{*} r^{*} \geq 0$ for $r^{*} \geq r_{0}$. Since $b_{r^{*}}^{*}<0, \frac{d K^{*}}{d t}$ will be positive where $\frac{d r^{*}}{d t}<0$ and where $1+\pi^{*} r^{*} \geq 0$. Using P6, part (b) follows.

Finally, let $\theta=f_{0}$. From P6, $\frac{d r^{*}}{d f_{0}}=0$ and $b^{*}$ does not explicitly depend on $f_{0}$. Furthermore, both $\frac{d f^{*}}{d f_{0}}>0$ and $b_{f^{*}}^{*}<0$, thus $\operatorname{sign} \frac{d K^{*}}{d f_{0}}<0$.

Proof of P8. As was shown in the text, $F=\frac{1-\left(1-K^{*}\right)^{2}}{2}$ when $b^{*}>0$ and 0 otherwise. As argued in the proof of $\mathrm{P} 2, b^{*}>0$ if $r^{*}<t /(1-t)$ and is negative otherwise. So

$$
F=\left\{\begin{array}{cc}
0 & \text { if } r^{*} \geq t /(1-t) \\
\frac{1-\left(1-K^{*}\right)^{2}}{2} & \text { if } r^{*}<t /(1-t)
\end{array} .\right.
$$

Differentiation with respect to $\theta, \theta=\delta, t$ or $f_{0}$ yields the result.

Proof of P9. Prom P8, we have that $\operatorname{sign} \frac{d F}{d \theta}=\operatorname{sign} \frac{d K^{*}}{d \theta}$. Using P7, the result follows.

\section{References}

*We are pleased to thank the participants in the Finance, Economics, and Center for Research Seminars at Essec Business School, the Centre for New and Emerging Markets Seminar at the London Business School, the Economics Seminar at the University of Sydney, the Economics Seminar at the University of Melbourne, and the IAES Warsaw conference for their helpful comments on earlier working papers that examined aspects of the problem we address here. The first version of the current paper was presented at the IAES 2011 Athens conference, and again, we are grateful for the useful comments. We also benefited from discussions with Patricia Charlety, Radu Vranceanu, and Francois Contensou, and the hospitality of Essec Business School. We thank Anatoly Garelik for extending the Kornai study of election turnovers. 
Acemoglu, Daron, Johnson, Simon and Robinson, James A., 2001. "The Colonial Origins of Comparative Development: An Empirical Investigation," American Economic Review 91, 1369-1401.

Acemoglu, Daron, Johnson, Simon and Robinson, James A., 2002. "Reversal of Fortune: Geography and Institutions in the Making of the Modern World Income Distribution," Quarterly Journal of Economics 117, 1231-1294.

Acemoglu, Daron, Johnson, Simon and Robinson, James A., 2003. "Disease and Development in Historical Perspective," Journal of the European Economic Association 1, 397-405.

Alexeev, Michael, Janeba, Eckhard and Osborne, Stefan, 2004. "Taxation and evasion in the presence of extortion by organized crime," Journal of Comparative Economics 32, 375-387.

Baker, Scott R., Bloom, Nicholas and Davis, Stephen J., 2012. "Measuring Economic Policy Uncertainty," http://policyuncertainty.com/media/BakerBloomDavis.pdf

Bevan, Alan A. and Estrin, Saul, 2004. "The Determinants of Foreign Direct Investment into European Transition Economies," Journal of Comparative Economics $32,775-787$.

Campos, Nauro F. and Coricelli, Fabrizio., 2002. "Growth in Transition: What We Know, What We Don't, and What We Should," Journal of Economic Literature 40: 3, 793-836.

Dixit, Avinash K., 2004. Lawlessness and Economics:Alternative Modes of Governance. Princeton: Princeton University Press.

Djankov, Simeon and Murrell, Peter, 2002. "Enterprise Restructuring in Transition, Journal of Economic Literature, 40, 739-792.

European Bank for Reconstruction and Development, Transition Reports, various years.

European Bank for Reconstruction and Development-World Bank Business Envi- 
ronment and Enterprise Performance Survey (BEEPS II), 2002.

Grossman, Herschel I., 1995. "Rival Kleptocrats: The Mafia Versus the State," in Fiorentini, Gianluca and Peltzman, Sam (Eds.), The Economics of Organized Crime, Cambridge: Cambridge University Press.

Hoff, Karla and Stiglitz, Joseph E., 2004 "After the Big Bang? Obstacles to the Emergence of the Rule of Law in Post-Communist Societies," American Economic Review, 94:3, 753-763.

Katz, Barbara G. and Owen, Joel, 2009. "Are Property Rights Enough? Reevaluating a Big-Bang Claim," Economies in Transition 17:1, 75-96.

Kornai, Janos, 2006. "The Great Transformation of Central Eastern Europe: Success and Disappointment," Economics of Transition, 14:2, 207-244

McMillan, John, 2002. Reinventing the Bazaar. New York: W. W. Norton.

Polishchuk, Leonid and Savvateev, Alexei, 2004. "Spontaneous (non)emergence of Property Rights," Economics of Transition, 12:1, 103-127.

Roland, Gérard and Verdier, Thierry (2003). "Law Enforcement and Transition,". European Economic Review, 47:4, 669-685

Shleifer, Andrei and Vishny, Robert W., 1998. The Grabbing Hand: Government Pathologies and Their Cures. Cambridge: Harvard University Press.

Sonin, Konstantin, 2003. "Why the Rich May Favor Poor Protection of Property Rights," Journal of Comparative Economics, 31, 715-731. 